Advance Journal of Food Science and Technology 17(2): 13-17, 2019

DOI:10.19026/ajfst.17.5997

ISSN: 2042-4868; e-ISSN: 2042-4876

(C) 2019 Maxwell Scientific Publication Corp.

Submitted: August 13, 2018

Accepted: October 17, 2018

Published: March 25, 2019

\title{
Research Article \\ Relationship between the Maillard Reaction and Lipid Peroxidation in Adding Various Fatty Acids on Bread Baking
}

\author{
${ }^{1}$ Toshiyuki Toyosaki, ${ }^{2}$ Michio Kasai and ${ }^{1}$ Yasuhide Sakane \\ ${ }^{1}$ Department of Foods and Nutrition, Koran Women's Junior College Fukuoka, 811-1311, Japan \\ ${ }^{2}$ Central Research Laboratory of the Nisshin OilliO Group LTD 1-Banchi, Shinmei-cho, Yokosuka \\ Kanagawa, 239-0832, Japan
}

\begin{abstract}
The Maillard reaction accompanying the baking of bread largely affects the quality of finished bread and the degree of taste. Particularly, the difference of lipids as a secondary material can be considered as one of the major factors. We examined how the differences in constituent fatty acids of lipids in secondary materials are related to Maillard reaction. Chemical differences in added fatty acids were identified to be a major factor in the induction of the Maillard reaction and lipid peroxidation in bread baking. With saturated fatty acids, a higher carbon number tended to promote the Maillard reaction induction and suppress lipid peroxidation induction. Conversely, with unsaturated fatty acids, a greater degree of unsaturation tended to suppress the Maillard reaction induction and promote lipid peroxidation induction. The present findings clarified the involvement of fatty acid carbon number and degree of unsaturation in the Maillard reaction and lipid peroxidation inductions in bread baking. This phenomenon is a novel finding for the food science field.
\end{abstract}

Keywords: Bread baking, lipid peroxidation, maillard reaction, medium-chain triacylglycerols, saturated fatty acids, unsaturated fatty acids

\section{INTRODUCTION}

Studies on Medium Chain Triacylglycerol (MCT) consisting only of medium chain fatty acids (C8 and C10) have been studied and during that time there have been numerous excellent research reports. However, most of them focus mostly on clinical nutrition or biochemical perspectives (Seaton et al., 1986; Lavau and Hashim, 1978; Geliebter et al., 1983; Chanez et al., 1991; Kris-Etherton and Yu, 1997; Kritchevsky and Tepper, 1965; Leveille et al., 1967; Ecelbarger et al., 1991; Papamandjaris et al., 1998). In contrast, very few studies have been conducted from a food science standpoint.

We have found interesting knowledge that the fermentation of the dough is enhanced as a result of tracking from a few years ago on the food function properties of MCT's bread and also clarified the fact that MCT suppresses the induction of Maillard reaction (Toyosaki, 2018). Based on such research results, MCT believes that new knowledge can be brought up on the food function characteristics of bread.

By the way, it is a well known problem in the food chemistry and biochemistry fields that oxidation of lipids in foods causes the formation of offensive odor and potentially hazardous compounds (Ames et al., 1993; Gardner, 1996; Pokorny, 1999; Shewfelt and Del Rosario, 2000; Mercier and Gelinas, 2001; Toyosaki and Sakane, 2002; Toyosaki and Koketsu, 2004; Grosch, 1987). It is considered that the lipid peroxidation reaction induced during the baking of bread has a great adverse effect on the flavor and texture of bread. In this study, it is extremely important to track the relationship between Maillard reaction and fatty acid, but no research report on it is found.

The objective of the current study was to investigate the relationship between the Maillard reaction and hydroperoxides produced by lipid peroxidation in bread baking promoting with various fatty acids and the mechanism that is involved.

\section{MATERIALS AND METHODS}

Materials: Materials were purchased from the following sources. Flour (Super King 13.8\%, Ash $0.42 \%$, Water $14 \%$ ) was obtained from Nisshin Seifun (Chiyoda Ward, Tokyo). Protein, ash, lipid and water content were $13.1 \%$ (Kjeldahl, N x 6.25), 0.4\%, 1.8\% and $15.0 \%$, respectively. More than $95 \%$ of the flour granules were sifted through a $132 \mathrm{~mm}$ mesh sieve. Dry

Corresponding Author: Toshiyuki Toyosaki, Department of Foods and Nutrition, Koran Women's Junior College Fukuoka, 811-1311, Japan, Tel.: +81-92-581-1538

This work is licensed under a Creative Commons Attribution 4.0 International License (URL: http://creativecommons.org/licenses/by/4.0/). 
yeast (Saccharomyces cerevisias) was purchased from S. I. Lesaffre (Marcq-en-Baroeul, France). Gluten (purity $>90 \%$ ). Glucose and lysine were purchased from Nakarai Tesque, Inc. (Kyoto, Japan). Wako Pure Chemical Ind. 95\% Chemotherapeutic agent (C8: 0, C12: 0, C16: 0, C18: 0, C18: 1, C18: 2, C20: 4, C22: 6; $95 \%$ Yes, it was obtained from Nacalai Tesque.

Preparation and baking of dough: Dough was prepared using commercially available ingredients to prepare bread dough and using straight dough method. More specifically, the dough was prepared using ingredients for preparing bread, i.e., lipid, strong flour, live yeast, water, sugar, salt and skimmed milk powder. The added lipid content was $10 \%$. The temperature of the dough at the completion of mixing was $26^{\circ} \mathrm{C}$. and the dough was fermented at a temperature of 28 to $30^{\circ} \mathrm{C}$. for $90 \mathrm{~min}$. To study the influence of fatty acids on bread baking of C8: 0, C12: 0, C16: 0 and C18: 0 saturated fatty acids and C18: 1, C18: 2, C20: 4 and C22: 6, unsaturated Fatty acids were used in the test. Subsequently, the dough was molded, finally fermented at a temperature of $36^{\circ} \mathrm{C}$. and a humidity of $75 \%$ for 60 to $70 \mathrm{~min}$ and then baked at $230^{\circ} \mathrm{C}$. for 35 to $40 \mathrm{~min}$ until $210^{\circ} \mathrm{C}$. (bottom of the oven). In this experiment, dough with only primary fermentation was also used.

Extraction of Advanced Glycation Endproducts (AGEs) from baked bread and determination of amount of AGEs: Using $50 \mathrm{~mL}$ of $0.25 \mathrm{M}$ phosphate buffer (pH 7.4), AGE was extracted from $100 \mathrm{~g}$ of the baked bread. AGEs are brown pigments and the absorbance at $420 \mathrm{~nm}$ (brown coloring) was measured.

Measurement of hydroperoxide: The hydroperoxide concentration was calculated with 2', 7'dichlorofluorescein (DCF). For demulsification, a 5.0 $\mathrm{ml}$ sample was centrifuged $(10,000 \times \mathrm{g}, 30 \mathrm{~min})$. Linoleic acid in the supernatant was then measured and hydroperoxide levels were measured using the method of Cathcart et al. (1984). First, 1.0 mL of 1.0 M DCF solution in ethanol and $2.0 \mathrm{ml}$ of $0.01 \mathrm{~N} \mathrm{NaOH}$ were mixed, stirred for 30 minutes and neutralized with 10 $\mathrm{mL}$ of $25 \mathrm{mM}$ phosphate buffer ( $\mathrm{pH}$ 7.2). Then a 2.0 $\mathrm{mM}$ neutralized DCF solution was added to a solution of hematin $(10 \mathrm{mM})$ in $25 \mathrm{mM}$ phosphate buffer $(\mathrm{pH}$ 7.2; $0.01 \mathrm{mg} \mathrm{DCF} / \mathrm{mL}$ ). $2.0 \mathrm{~mL}$ of the hematin-DCF solution and $10 \mathrm{~mL}$ of the linoleic acid sample were mixed and allowed to stand at $50^{\circ} \mathrm{C}$. for $50 \mathrm{~min}$, followed by fluorescence measurement (excitation at $400 \mathrm{~nm}$, emission at $470 \mathrm{~nm}$ ) and DCF was measured. This method measures hydroperoxides with higher sensitivity than the iron rhodanide method commonly used.

Statistical analysis: All data were shown as mean \pm standard deviation. Statistical comparisons between different treatments were approved using Duncan's new multi-range test (Steel and Torrie, 1980) and application software Stat View 512 (Statistical Information Brain Power, Berkeley, Calif., USA) It was done by $t$ test. The significance level was set at $\mathrm{p}<0.05$ in all statistical tests.

\section{RESULTS AND DISCUSSION}

Changes in the Maillard reaction and lipid peroxidation inductions during baking of dough with different added saturated fatty acids: Figure 1 and 2 show the effects of different saturated fatty acids on the Maillard reaction and changes in lipid peroxidation, respectively, induced up to $1 \mathrm{~h}$ of baking bread dough. An increase in the carbon number of the added saturated fatty acids tended to increase the Maillard reaction induction, while conversely suppressing lipid peroxidation induction.

Products of the Maillard reaction have known antioxidant effects; thus, they suppress lipid peroxidation (Nasrollahzadeh et al., 2017; Dong et al., 2012). Therefore, the following inferences can be made from the present findings. The lower the carbon number of the added saturated fatty acids, the greater the promotion of the Maillard reaction, leading to greater quantities of the products of this reaction (primarily melanoidins) and a more marked antioxidant effect in the form of decreased lipid peroxidation.

Effects of changes in saturated fatty acid carbon number on the Maillard reaction and lipid peroxidation: Figure 3 shows the relationships
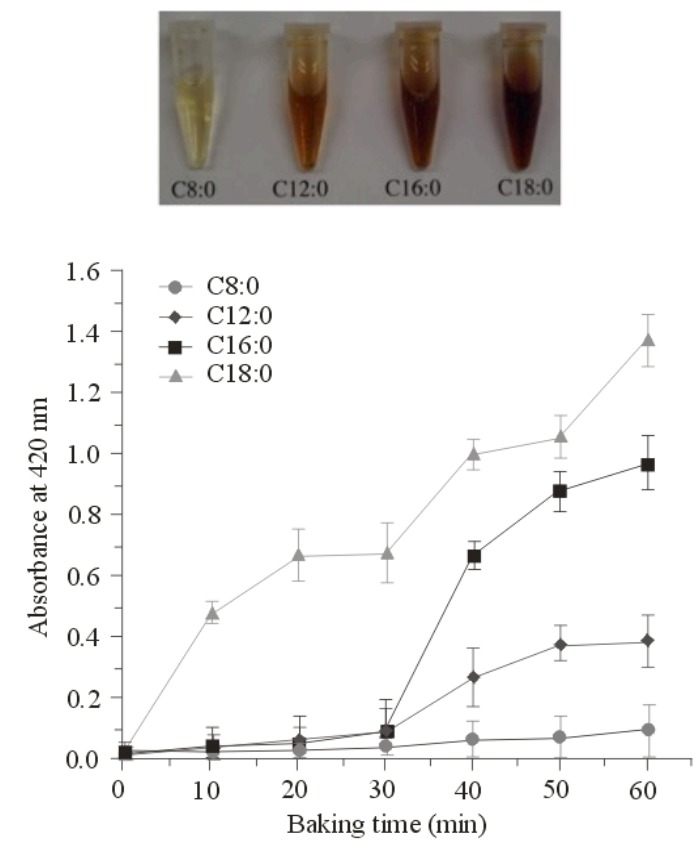

Fig. 1: Changes in AGEs formation for the different saturated fatty acids 


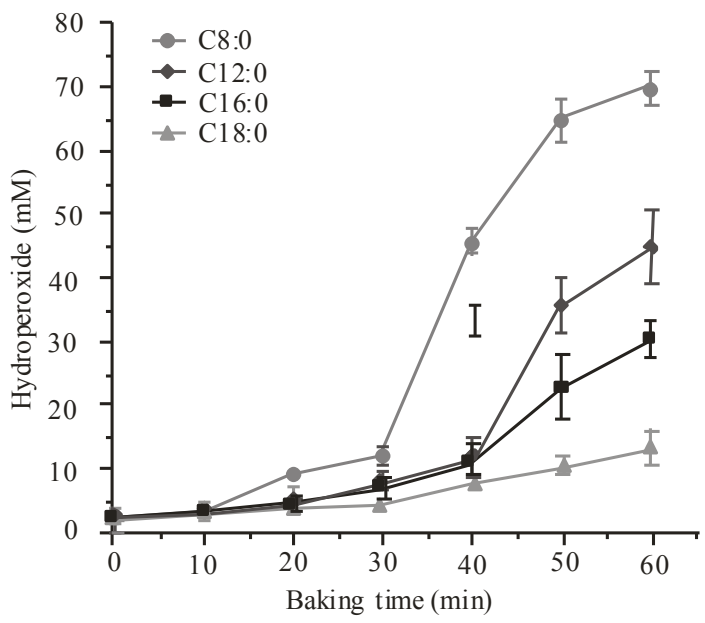

Fig. 2: Changes in hydroperoxides for the different saturated fatty acids

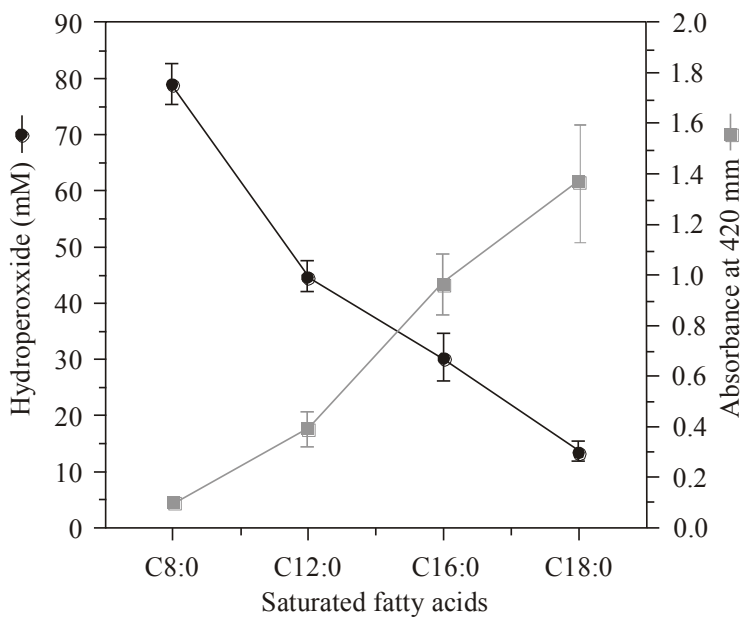

Fig. 3: Relationship between the Maillard reaction and the lipid peroxidation on the bread baking with different saturated fatty acids

between changes in the carbon number of different added saturated fatty acids and the Maillard reaction and lipid peroxidation. The higher the fatty acid carbon number, the greater the promotion of the Maillard reaction induction and the tendency toward suppression of lipid peroxidation induction. To our knowledge, the present study is the first to report this phenomenon, which has major significance for the bread industry. Further investigation of the mechanisms of this phenomenon is required.

Changes in the Maillard reaction and lipid peroxidation inductions during baking of dough with different added unsaturated fatty acids: Figure 4 and 5 show the effects of different unsaturated fatty acids on the Maillard reaction and changes in lipid peroxidation, respectively, induced up to $1 \mathrm{~h}$ of baking bread dough. An increase in the degree of unsaturation of added unsaturated fatty acids tended to increase the
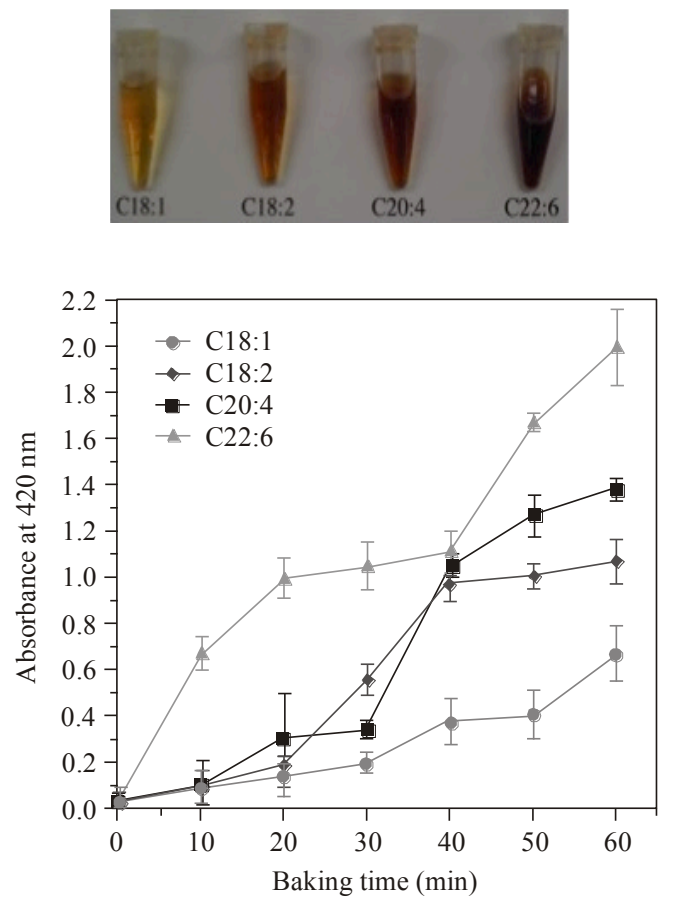

Fig. 4: Changes in AGEs formation for the different unsaturated fatty acids

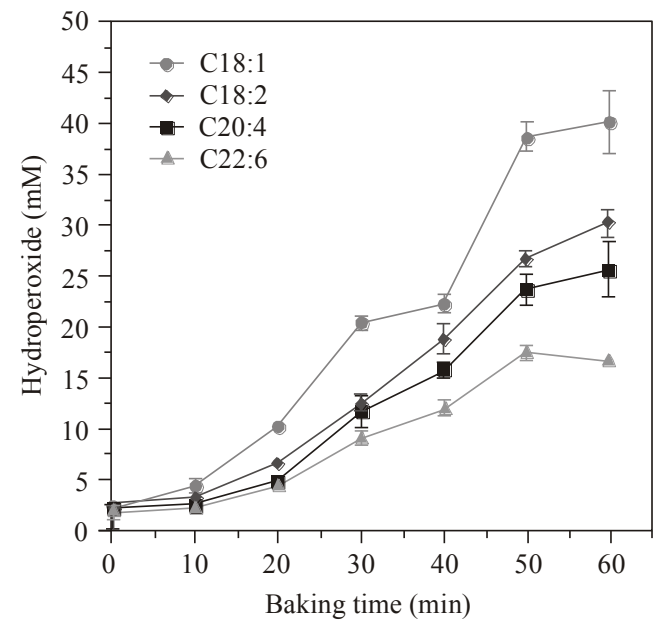

Fig. 5: Changes in hydroperoxides for the different unsaturated fatty acids

Maillard reaction induction while suppressing lipid peroxidation induction. These findings differ completely from those regarding saturated fatty acids, suggesting the existence of a mechanism by which changes in the degree of unsaturation has a greater effect than changes in the carbon number on the Maillard reaction and lipid peroxidation inductions when unsaturated fatty acids are used.

Effects of changes in the degree of unsaturation of unsaturated fatty acids on the Maillard reaction and lipid peroxidation: Figure 6 shows the relationships 


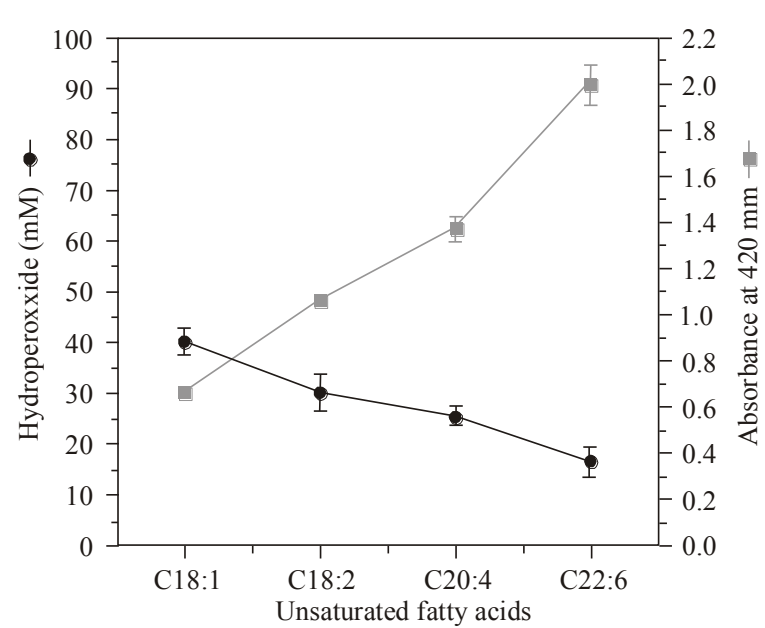

Fig. 6: Relationship between the Maillard reaction and the lipid peroxidation on the bread baking with unsaturated fatty acids

between changes in the degree of unsaturation of different added unsaturated fatty acids and the Maillard reaction and lipid peroxidation inductions. The greater the degree of unsaturation of the unsaturated fatty acids, the greater the tendency toward suppression of the Maillard reaction induction and promotion of lipid peroxidation induction. Clarification of this phenomenon represents a major contribution for the bread industry and the entire food science field.

\section{CONCLUSION}

The present findings demonstrate that differences in the chemical structure of different fatty acids greatly affect bread baking. With saturated fatty acids, changes in the carbon number had a marked effect on the Maillard reaction and lipid peroxidation inductions. Conversely, with unsaturated fatty acids, changes in the degree of unsaturation had a greater influence than carbon number on the Maillard reaction and lipid peroxidation inductions. The present findings represent a major contribution for the bread industry and the entire food science field.

\section{REFERENCES}

Ames, B.M., M.K. Shigena and T.M. Hagen, 1993. Oxidants, antioxidants and the degenerative diseases of aging. P. Natl. Acad. Sci. USA, 90(17): 7915-7922.

Cathcart, R., E. Schwiers and B.N. Ames, 1984. Detection of picomole levels of lipid hydroperoxides using a dichlorofluorescein fluorescent assay. Methods Enzymol., 105: 352-8.

Chanez, M., B. Bois-Joyeux, M.J. Arnaud and J. Peret, 1991. Metabolic effects in rats of a diet with a moderate level of medium-chain triglycerides. J. Nutr., 121(5): 585-594.
Dong, S., A. Panya, M. Zeng, B. Chen, D. Juilan McClements et al., 2012. Characteristics and antioxidant activity of hydrolyzed $\beta$-lactoglobulinglucose Maillrad reaction products. Food Res. Int., 46(01): 55-61.

Ecelbarger, G.L., J.B. Lasekan and D.M. Ney, 1991. In vivo triglyceride secretion and hepatic and plasma lipids in rats fed medium-chain triglycerides, tripelargonin, or corn oil. J. Nutr. Biochem., 2(5): 260-266.

Gardner, H.W., 1996. Lipoxygenase as a versatile biocatalyst. J. Am. Oil Chem. Soc., 73(11): 13471350.

Geliebter, A., N. Torbay, E.F. Bracco, S.A. Hashim and T.B. Van Itallie, 1983. Overfeeding with mediumchain triglyceride diet results in diminished deposition of fat. Am. J. Clin. Nutr., 37(1): 1-4.

Grosch, W., 1987. Reactions of Hydroperoxidesproducts of Low Molecular Weight. In: Chan, H.W.S. (Ed.), Autoxidation of Unsaturated Lipids. Academic Press Inc., London.

Kris-Etherton, P.M. and S. Yu, 1997. Individual fatty acid effects on plasma lipids and lipoproteins: Human studies. Am. J. Clin. Nutr., 65(5 Suppl): 1628S-1644S.

Kritchevsky, D. and S.A. Tepper, 1965. Influence of Medium-Chain Triglyceride (MCT) on cholesterol metabolism in rats. J. Nutr., 86(1): 67-72.

Lavau, M.M. and S.A. Hashim, 1978. Effect of medium chain triglyceride on lipogenesis and body fat in the rat. J. Nutr., 108(4): 613-620.

Leveille, G.A., R.S. Pardini and J.A. Tillotson, 1967. Influence of medium-chain triglycerides on lipid metabolism in the rat. Lipids, 2(4): 287-294.

Mercier, M. and P. Gelinas, 2001. Effect of lipid oxidation on dough bleaching. Cereal. Chem., 78(1): 36-38.

Nasrollahzadeh, F., M. Varidi, A. Koocheki and F. Hadizadeh, 2017. Effect of microwave and conventional heating on structural, functional and antioxidant properties of bovine serum albuminmaltodextrin conjugates through Maillard reaction. Food Res. Int., 100: 289-297.

Papamandjaris, A.A., D.E. Macdougall and P.J. Jones, 1998. Medium chain fatty acid metabolism and energy expenditure: Obesity treatment implications. Life Sci., 62(14): 1203-1215.

Pokorny, J., 1999. Handbook of Food Preservation, Antioxidants in Food Preservation. Marcel Dekker, New York.

Seaton, T.B., S.L. Welle, M.K. Warenko and R.G. Campbell, 1986. Thermic effect of medium-chain and long-chain triglycerides in man. Am. J. Clin. Nutr., 44(5): 630-634.

Shewfelt, R.L. and B.A. Del Rosario, 2000. The role of lipid peroxidation in storage disorders of fresh fruits and vegetables. HortScience, 35(4): 575-579. 
Steel, R.G.D. and J.H. Torrie, 1980. Principles and Procedures of Statistics: A Biometrical Approach. 2nd Edn., McGraw-Hill, New York.

Toyosaki, T. and Y. Sakane, 2002. Antioxidant effect of $\mathrm{NaCl}$ on the aqueous solution, emulsified, and enzymic lipid peroxidation. Bull. Soc. Sea Water Sci. Jpn., 56: 10-16.
Toyosaki, T. and M. Koketsu, 2004. Oxidative stability of silky fowl eggs. Comparison with hen eggs. J. Agr. Food Chem., 52(5): 1328-1330.

Toyosaki, T., 2018. Effects of medium-chain triacylglycerols on Maillard reaction in bread baking. J. Sci. Food Agr., 98(8): 3169-3174. 